\title{
Identification of Efficient Subgame-Perfect Nash Equilibria in a Class of Differential Games ${ }^{1}$
}

\author{
J. P. Rincón-Zapatero, ${ }^{2}$ G. Martín-Herrán, ${ }^{2}$ and J. Martínez ${ }^{2}$
}

Communicated by M. Simaan

\begin{abstract}
We present a method for the characterization of subgameperfect Nash equilibria being Pareto efficient in a class of differential games. For that purpose, we propose a new approach based on new necessary and sufficient conditions for computing subgame-perfect Nash equilibria.
\end{abstract}

Key Words. Differential games, subgame-perfect Nash equilibria, Pareto optimum, quasilinear partial differential equations.

\section{Introduction}

In the economic literature, there is a large number of papers where interactions between the decisions of the economic agents arise. One interesting subject is when these interactions can lead to an efficient solution, without the consideration of binding agreements between the agents.

In Ref. 1, Friedman states that it is not known whether the noncooperative outcome is Pareto efficient. The aim of this paper is to establish necessary and sufficient conditions giving an a priori characterization of the Pareto efficiency of feedback Nash equilibria for differential games with unidimensional state and control variables and where each control is a

\footnotetext{
${ }^{1}$ The authors thank the anonymous referees for helpful comments.

${ }^{2}$ Assistant Professor, Departamento de Economía Aplicada (Matemáticas), Facultad de Ciencias Económicas, Universidad de Valladolid, Valladolid, Spain.
} 
smooth function of the state and time variables. To this end, we propose a new approach based on the characterization of feedback Nash equilibria as solutions to a system of quasilinear partial differential equations as shown in Ref. 2. It is well known that, in general, the use of feedback strategies in noncooperative games prevents the attainment of efficient outcomes. To our knowledge, there are not necessary and sufficient conditions in the literature to be applied to general differential game models that allow the determination of whether the feedback Nash equilibrium is or is not a Pareto optimum. This paper is devoted to establishing this type of conditions.

\section{Game Description and Characterization of Subgame-Perfect Nash Equilibria}

We consider a two-person nonzero-sum differential game over a fixed time interval,

$$
\begin{array}{ll}
\max _{u^{i}} & \left\{J^{i}\left(t_{0}, x_{0}, u^{1}, u^{2}\right)=\int_{t_{0}}^{t_{f}} L^{i}\left(t, x, u^{1}, u^{2}\right) d t+S^{i}\left(t_{f}, x\left(t_{f}\right)\right)\right\}, \\
\text { s.t. } & \dot{x}=f\left(t, x, u^{1}, u^{2}\right), x\left(t_{0}\right)=x_{0}, \\
& u^{i}(t) \in U^{i}, \quad \forall t \in\left[t_{0}, t_{f}\right], U^{i} \text { an open subset of } \mathbb{R},
\end{array}
$$

for $i=1,2$. Here, the functions $L^{i}, f, S^{i}$ are assumed to be twice continuously differentiable. We denote by $x$ the state variable of the game, and $u^{i}$ represents the control variable for the $i$ th player. We associate to each $u^{i}$ a smooth function $\phi^{i}:\left[t_{0}, t_{f}\right] \times \mathbb{R} \rightarrow U^{i}$ such that $u^{i}(t)=\phi^{i}(t, x(t))$. We denote by $U^{i}$ the set of functions $\phi^{i}$ and $U=U^{1} \times U^{2}$.

The system

$$
\dot{x}=f\left(t, x, \phi^{1}(t, x), \phi^{2}(t, x)\right), \quad x\left(t_{0}\right)=x_{0},
$$

is the closed-loop system associated to the pair of feedback strategies $\left(\phi^{1}, \phi^{2}\right)$.

We remit the reader to Ref. 3 for the definitions of subgame-perfect Nash equilibrium and Pareto optimum.

In this note, we use a method proposed in Ref. 2 for computing Nash equilibria in feedback strategies. This method gives necessary and sufficient conditions characterizing subgame-perfect equilibria by means of the 
following system of quasilinear partial differential equations:

$$
\begin{aligned}
H_{u^{i} t}^{i_{i}}+H_{u^{i} x}^{i_{i}} f+\sum_{j=1}^{2} H_{u^{i} u^{j}}^{i^{j}}\left(\phi_{t}^{j}+\phi_{x}^{j} f\right)+H_{u^{i} \lambda^{i}}^{i^{i}}\left(-H_{x}^{i}-\sum_{j=1}^{2} H_{u^{j}}^{i} \phi_{x}^{j}\right) & =0, \\
i, j & =1,2,
\end{aligned}
$$

where $H^{i}\left(t, x, u^{1}, u^{2}, \lambda^{i}\right)$ is the Hamiltonian function associated to the $i$ th player, defined by

$$
H^{i}\left(t, x, u^{1}, u^{2}, \lambda^{i}\right)=L^{i}\left(t, x, u^{1}, u^{2}\right)+\lambda^{i} f\left(t, x, u^{1}, u^{2}\right),
$$

with $\lambda^{i}$ the costate variable. In Eq. (4), $\phi^{1}, \phi^{2}$ are the dependent variables and $t, x$ the independent variables.

The boundary condition over the costate variable established by the maximum principle and the expression obtained from the maximization of the Hamiltonian function provide a complete set of final conditions for the system (4) given by

$$
L_{u^{i}}^{i}\left(t_{f}, x, \phi^{1}, \phi^{2}\right)+f_{u^{i}}\left(t_{f}, x, \phi^{1}, \phi^{2}\right) S_{x}^{i}\left(t_{f}, x\right)=0, \quad i=1,2 .
$$

In Theorem 4.1 of Ref. 2, we show that, under suitable hypotheses about the Hamiltonian functions, a classical solution $\hat{\phi}$ of system (4) becomes a subgame-perfect Nash equilibrium of the differential game. That is, this system gives a set of conditions not only necessary, but also sufficient for optimality. Furthermore, in the infinite-horizon case, the condition

$$
\lim _{t \rightarrow \infty}(\partial y / \partial x)(t)\left(-f_{u^{i}}^{-1} L_{u^{i}}^{i}\right)(t, y(t), \hat{\phi}(t, y(t))=0, \quad i=1, \ldots, N,
$$

where $\dot{y}=f(s, y, \hat{\phi})$ and $y(t)=x$, implies that $\hat{\phi}$ is a subgame-perfect Nash equilibrium.

\section{Necessary and Sufficient Conditions for Pareto Efficiency of a Subgame-Perfect Nash Equilibrium}

From now on, we center our attention on the statement of the necessary and sufficient conditions for Pareto efficiency of subgame-perfect Nash equilibrium.

Theorem 3.1. Let $\hat{\phi} \in U$ be a smooth subgame-perfect Nash equilibrium of the game (1)-(3), with $f_{u^{i}}(t, x, \hat{\phi}) \neq 0$. Let $H^{i}$ be of class $C^{2}$ with respect to $\left(u^{1}, u^{2}\right) \in \bigcup$ for all $t \in\left[0, t_{f}\right]$ and $x \in \mathbb{R}$. If $\hat{\phi}$ is Pareto optimum, then there exists $i \in\{1,2\}$ such that, for all $j \in\{1,2\}, j \neq i$,

$$
\left.\left(L_{u^{j}}^{i} f_{u^{i}}-L_{u^{i}}^{i} f_{u^{j}}\right)\right|_{(t, x, \hat{\phi})}=0, \quad \text { for all } t \in\left[t_{0}, t_{f}\right], x \in \mathbb{R} .
$$

Proof. See Ref. 4. 
Remark 3.1. The concavity of the Hamiltonian $H^{i}$ with respect to variables $\left(u^{1}, u^{2}\right)$ guarantees that the necessary condition (7) for the $i$ th player is sufficient too. If condition (7) is satisfied for $i=1,2$, together with the supplementary hypothesis of the Hamiltonians $H^{i}, i=1,2$, being concave with respect to all the control variables for all $t, x$, then the subgameperfect Nash equilibrium is an absolutely cooperative solution. On the other hand, let us observe that condition (7) is fulfilled for zero-sum games and team problems.

Our purpose now is to establish sufficient conditions for Pareto efficiency of a subgame-perfect Nash equilibrium. We denote by $u^{2}=$ $\Theta\left(t, x, u^{1}\right)$ the necessary relation (7) between the strategies of the two players. By replacing this relation in (4), we obtain an overdetermined system. We look for conditions ensuring the existence of solutions to this system. To this end, we introduce the following notation for the two partial differential equations that arise from (4) once we have substituted $u^{2}=\Theta\left(t, x, u^{1}\right)$ :

$$
F_{i}\left(t, x, u^{1}, p, q\right)=a_{i}\left(t, x, u^{1}\right) p+b_{i}\left(t, x, u^{1}\right) q+c_{i}\left(t, x, u^{1}\right), \quad i=1,2 .
$$

Here,

$$
p=u_{t}^{1}, \quad q=u_{x}^{1},
$$

and

$$
\begin{aligned}
& a_{i}=H_{u^{i} u^{i}}^{i^{i}}+H_{u^{i} u^{j}}^{i^{j}} \Theta_{u}, \\
& b_{i}=H_{u^{i} u^{i}}^{i^{i}} f+H_{u^{i} u^{j}}^{\Theta^{i}} \Theta_{u} f-f_{u^{i}} H_{u^{j}}^{i} \Theta_{u}, \\
& c_{i}=H_{u^{i} t}^{i^{i}}+H_{u^{i} x}^{i^{i}} f+H_{u^{i} u^{j}}^{i^{j}}\left(\Theta_{t}+\Theta_{x} f\right)-f_{u^{i}}\left(H_{x}^{i}+H_{u^{j}}^{i} \Theta_{x}\right) .
\end{aligned}
$$

All functions are evaluated at $\left(t, x, u^{1}, \Theta\left(t, x, u^{1}\right)\right)$ and $\lambda^{i}=-f_{u^{i}}^{-1} L_{u^{i}}^{i}$. Assuming that

$$
\Delta=\left|\begin{array}{ll}
a_{1} & b_{1} \\
a_{2} & b_{2}
\end{array}\right| \neq 0, \quad \text { for all } t, x, u^{1}
$$

then

$$
\left[\begin{array}{l}
p \\
q
\end{array}\right]=\left[\begin{array}{ll}
a_{1} & b_{1} \\
a_{2} & b_{2}
\end{array}\right]^{-1}\left[\begin{array}{l}
-c_{1} \\
-c_{2}
\end{array}\right] .
$$

We denote by $\left[F_{1}, F_{2}\right]$ the expression

$$
\begin{aligned}
\partial\left(F_{1}, F_{2}\right) / \partial(x, q) & +p\left(\partial\left(F_{1}, F_{2}\right) / \partial\left(u^{1}, p\right)\right) \\
& +\partial\left(F_{1}, F_{2}\right) / \partial(t, p)+q\left(\partial\left(F_{1}, F_{2}\right) / \partial\left(u^{1}, q\right)\right),
\end{aligned}
$$

once we have replaced $p, q$ by their expressions given by (8). 
Theorem 3.2. Suppose that the following conditions are satisfied:

(C1) The pair $\left(\hat{\phi}^{1}, \Theta\right)$ is a smooth feedback Pareto optimum of problem (1)-(3), such that $f_{u^{i}}\left(t, x, \hat{\phi}^{1}, \Theta\right) \neq 0$.

(C2) $\left[F_{1}, F_{2}\right]=0$.

(C3) The pair $\left(\hat{\phi}^{1}, \Theta\right)$ satisfies the final condition required in (5) associated to problem (1)-(3).

(C4) For all $u^{1} \in U^{1}, u^{2} \in U^{2}, t \in\left[0, t_{f}\right], x \in \mathbb{R}$, $H^{1}\left(t, x, \hat{\phi}^{1}, \Theta, \Gamma^{1}\right) \geq H^{1}\left(t, x, \hat{\phi}^{1}, u^{2}, \Gamma^{1}\right)$, $H^{2}\left(t, x, \hat{\phi}^{1}, \Theta, \Gamma^{2}\right) \geq H^{2}\left(t, x, u^{1}, \Theta, \Gamma^{2}\right)$, where $\Gamma^{i}$ equals $-f_{u^{\prime}}^{-1} L_{u^{i}}^{i}$ evaluated at $\left(t, x, \hat{\phi}^{1}, \Theta\right), i=1,2$.

Then, $\left(\hat{\phi}^{1}, \Theta\right)$ is a subgame-perfect Nash equilibrium.

Proof. See Ref. 4.

Remark 3.2. As we did in Remark 2.1, when an infinite horizon is considered, the final condition (C3) in Theorem 3.2 must be replaced by the sufficient transversality condition (6).

Remark 3.3. When $\Delta \neq 0$ and the compatibility condition (C2) stated in Theorem 3.2 is not fulfilled, then the Pareto optimum cannot be a Nash equilibrium. In fact, $\left[F_{1}, F_{2}\right]=0$ is a necessary condition for efficiency of the Nash equilibrium. This provides a negative criterion based on a full computational method. When $\Delta=0$ and the equations $F_{1}=0, F_{2}=0$ are not such that one implies the other, then there is no common solution to the equations.

\section{Application to a Monetary and Fiscal Regulation Differential Game}

We are aware that the efficiency of Nash equilibrium is a very rare property; in fact, there are few examples in the economic literature. However, this property can be found in the familiar linear-quadratic differential games, as the following example shows.

Example 4.1. We consider a two-person scalar linear-quadratic differential game as proposed and analyzed in Ref. 5. In this model, the government debt accumulation $\dot{d}$ is the sum of interest payments on government debt $r d$ plus primary fiscal deficits $f$ minus seignorage $m$,

$$
\dot{d}=r d+f-m, \quad d(0)=d_{0} .
$$


In that paper, the authors assume that fiscal and monetary policies are controlled by the fiscal authority and the monetary authority, respectively, with different objectives. The players wish to minimize the deviations from fixed targets $\bar{f}, \bar{m}, \bar{d}$. We suppose that the two institutions wish a balanced budget, that is to say,

$$
r \bar{d}+\bar{f}-\bar{m}=0 .
$$

In this case, the game can be formulated as follows:

$$
\begin{aligned}
& \max _{u^{1}}(1 / 2)\left\{\int_{0}^{t_{f}} \exp \left(-\delta_{1} t\right)\left(-\left(u^{1}\right)^{2}-\eta\left(u^{2}\right)^{2}-\lambda x^{2}\right) d t\right\}, \\
& \max _{u^{2}}(1 / 2)\left\{\int_{0}^{t_{f}} \exp \left(-\delta_{2} t\right)\left(-\left(u^{2}\right)^{2}-\kappa x^{2}\right) d t\right\}, \\
& \text { s.t. } \quad \dot{x}=r x+u^{1}-u^{2}, \quad x(0)=d_{0}-\bar{d}, \\
& \text { where } \quad x=d-\bar{d}, \quad u^{1}=f-\bar{f}, \quad u^{2}=m-\bar{m},
\end{aligned}
$$

and $\eta, \lambda, \kappa$ are positive constants. Condition (7) for the first player implies

$$
u^{2}=\Theta\left(u^{1}\right)=(-1 / \eta) u^{1} .
$$

System (4) satisfying the above relation between the strategies of the two players reads as follows:

$$
\begin{aligned}
& u_{t}^{1}+u_{x}^{1}\left((1+1 / \eta) u^{1}+r x\right)=\left(\delta_{1}-r\right) u^{1}+\lambda x, \\
& u_{t}^{1}+u_{x}^{1}\left((2+1 / \eta) u^{1}+r x\right)=\left(\delta_{2}-r\right) u^{1}+\eta \kappa x .
\end{aligned}
$$

It can be shown that there is no common solution to (9)-(10) satisfying the final condition $u^{1}\left(t_{f}, x\right)=0$ in the finite-horizon game. For that reason, we center on the infinite-horizon case because there is no necessary transversality condition for optimal strategies. Since the problem is autonomous, stationary strategies are considered.

In order to test if there is some solution to system (9)-(10) satisfying the above relation between the strategies of the two players, we make use of the compatibility condition (C2). For this game, it appears as a homogeneous polynomial of degree two in the variables $x, u^{1}$, namely,

$$
a_{1} x^{2}+a_{2} x u^{1}+a_{3}\left(u^{1}\right)^{2} .
$$

There are only two possible cases.

Case 1. We assume that the identity

$$
\lambda-\kappa \eta=0
$$


holds. This condition and $a_{3}=0$ imply that all the coefficients of the polynomial are zero. It could be proved that there are no common linear solutions to Eqs. (9)-(10). Therefore, the compatibility condition guarantees the existence of at least one solution of Eqs. (9)-(10) simultaneously, that is nonlinear in this case. Nevertheless, for this kind of solutions, the fulfilment of the transversality condition (6) is not guaranteed. Therefore, we cannot assure that the solution is a Pareto efficient Nash equilibrium.

Case 2. When

$$
\lambda-\kappa \eta \neq 0,
$$

but

$$
\delta_{1}=\delta_{2}=\delta=2 r \quad \text { and } \quad \lambda+2 \lambda \eta-\kappa \eta-\kappa \eta^{2}=0,
$$

the polynomial is identically null. When $\eta \kappa-\lambda>0$, the common solutions independent of time of Eqs. (9)-(10) are

$$
u^{1}(x)_{ \pm}= \pm(\sqrt{\eta \kappa-\lambda})|x|,
$$

and the unique strategy that can make stable the dynamics is

$$
u^{1}(x)=-(\sqrt{\eta \kappa-\lambda}) x .
$$

Condition (6) is verified if

$$
r-(1+1 / \eta) \sqrt{\eta \kappa-\lambda}<0 .
$$

The concavity of $H^{i}$ with respect to his or her own control variable $u^{i}$ is ensured. As we pointed out in Remark 4.1 in Ref. 2, this assures the fulfillment of (C4) of Theorem 3.2. Moreover, the Hamiltonians $H^{1}$ and $H^{2}$ are strictly concave in the variables $\left(u^{1}, u^{2}\right)$ and $u^{2}$, respectively, that makes applicable Remark 3.1, assuring the Pareto optimality of the pair $\left(u^{1},(-1)\right.$ $\left.\eta) u^{1}\right)$. Consequently, all the conditions listed in Theorem 3.2 are fulfilled.

Even though we have shown our method for a linear-quadratic differential game, let us note that our technique applies to more general models. The method presents a very useful computational advantage that allows one to handle nonlinear models.

\section{References}

1. Friedman, A., Differential Games, Handbook of Game Theory, Edited by R. J. Aumann and S. Hart, North Holland, Amsterdam, Holland, Vol. 2, pp. 782-799, 1994. 
2. Rincón-Zapatero, J. P., Martínez, J., and Martín-Herrán, G., New Method to Characterize Subgame-Perfect Nash Equilibria in Differential Games, Journal of Optimization Theory and Applications, Vol. 96, pp. 377-395, 1998.

3. Leitmann, G., Cooperative and Noncooperative Many-Players Differential Games, Springer Verlag, New York, NY, 1974.

4. Rincón-Zapatero, J. P., Martin-Herrán, G., and Martinez, J., Characterization of Subgame-Perfect Nash Equilibria with the Property of Pareto Efficiency, Preprint, Universidad de Valladolid, Valladolid, Spain, 1998.

5. Aarle, B., Bovenberg, L., and Raith, M., Monetary and Fiscal Policy Interaction and Government Debt Stabilization, Journal of Economics, Vol. 62, pp. 111140, 1995. 